\title{
PROMOCIÓN DE LA SALUD Y PREVENCIÓN DE LAS ENFERMEDADES PARA UN ENVEJECIMIENTO ACTIVO Y CON CALIDAD DE VIDA
}

\author{
Elizabeth Aliaga-Díaz ${ }^{1,2, a}$, Sofía Cuba-Fuentes ${ }^{2,3, b}$, Marcela Mar-Meza $4,5, a$
}

\begin{abstract}
RESUMEN
Los adultos mayores representan un grupo poblacional en incremento, su estado de salud depende de muchos factores como el físico, cognoscitivo y sociofamiliar; por otro lado, tienen características como la heterogeneidad, la gran carga de enfermedad, la pluripatología, entre otras, que repercuten no solo en el ámbito familiar sino social. Es importante ofrecer a la población adulta mayor los medios para ejercer un mejor control sobre su salud y mejorarla. Todo ello con el fin de lograr un envejecimiento exitoso, es decir, sin discapacidad, con la menor cantidad de enfermedades posibles o adecuadamente controladas y, sobre todo, intentando mantener en todo momento su autonomía y calidad de vida, respetando siempre sus valores y preferencias. Por otro lado, las actividades preventivas en los adultos mayores deben tener en cuenta no solo el riesgo de enfermedad, sino también la alteración de la función que produce la enfermedad, así como aquellas condiciones frecuentes en el adulto mayor que pueden deteriorar su estado de salud como la fragilidad, las caídas, y las complicaciones iatrogénicas; las actividades preventivas en el adulto mayor deben estar dirigidas a todos estos factores. Presentamos algunas pautas que podrían ser importantes para promover un envejecimiento activo y actividades preventivas que podrán ser aplicadas según el contexto particular de cada persona.
\end{abstract}

Palabras clave: adulto mayor, envejecimiento activo, promoción de salud, prevención de salud. (fuente: DeCS BIREME).

\section{HEALTH PROMOTION AND DISEASE PREVENTION FOR ACTIVE AGING THAT PRESERVES QUALITY OF LIFE}

\begin{abstract}
Older adults represent a growing population whose health status depends on many factors, including physical, cognitive, and social factors, as well as family. They also have features including heterogeneity, a high disease burden, and comorbidities that affect the family and social spheres. It is important to offer the older adult population methods to exercise better control over their health and, thus, improve it. The goal is to achieve successful aging, that is, aging without disabilities, with the fewest possible or adequately controlled ailments while helping them maintain their autonomy and quality of life and always respecting their values and preferences. On the other hand, preventive activities in older adults consider the risk of disease; functional alteration causing the disease; and conditions common to the elderly that can damage their health, including frailty, falls, and iatrogenic complications. Preventive activities for the elderly should consider all of these factors. Here we present some guidelines that may be important for promoting active aging as well as preventive activities that can be used according to each person's individual situation.
\end{abstract}

Keywords: elderly, active aging, health promotion, health prevention. (source: MeSH NLM).

\section{INTRODUCCIÓN}

La Organización Mundial de Salud (OMS) definió la salud en 1948 como: "Un estado de completo bienestar físico, mental y social, y no solamente la ausencia de afecciones o enfermedades" (1). La carta de Otawa en 1986 definió la promoción de salud como: "Proceso que da a la población los medios para ejercer un mayor control sobre su propia salud y de mejorarla" (2). Los adultos mayores de hoy, tienen una expectativa de vida de casi 10 años más, y muchos de ellos requerirán actividades de promoción y prevención de salud que sean ofrecidas por el sistema de salud al que consultan. Desde la perspectiva de lograr un envejecimiento

\footnotetext{
Instituto de Gerontología. Universidad Peruana Cayetano Heredia. Lima, Perú.

Universidad Peruana Cayetano Heredia. Lima, Perú

Policlínico Juan José Rodríguez Lazo EsSalud. Lima, Perú.

Instituto Peruano de Neurociencias. Lima, Perú.

Hospital Central Fuerza Aérea del Perú. Lima, Perú.

Médico geriatra; ${ }^{\mathrm{b}}$ médico familiar

Recibido: 07/03/2016 Aprobado:04/05/2016
}

Citar como: Aliaga-Diaz E, Cuba-Fuentes S, Mar-Meza M. Promoción de la salud y prevención de las enfermedades para un envejecimiento activo y con calidad de vida. Rev Peru Med Exp Salud Publica. 2016;33(2)311-20. doi: 10.17843/rpmesp.2016.332.2143 
exitoso, sin discapacidad, con la menor cantidad de enfermedades posibles o adecuadamente controladas, y manteniendo autonomía. Consideramos que los siguientes puntos podrían ser importantes para el logro de este objetivo.

\section{PROMOCIÓN DE SALUD HACIA UN ENVEJECIMIENTO ACTIVO}

\section{INCORPORANDO LA PROMOCIÓN DE LA SALUD EN LA ATENCIÓN DE LOS ADULTOS MAYORES}

La promoción de salud puede ser abordada desde diferentes escenarios ${ }^{(3)}$. El escenario macrosocial, con enfoque en los determinantes sociales, debe ser abordado desde políticas públicas que disminuyan la inequidad en esta etapa de vida, donde suele haber mayor vulnerabilidad. El escenario microambiental, relacionado a los escenarios donde los adultos mayores se desenvuelven: casas, barrios, municipios; se puede influir a través de la concertación con gobiernos locales y otros actores de las localidades donde viven los adultos mayores. El escenario interpersonal, donde la familia es la principal fuente de apoyo social y de promoción de estilos de vida saludable, se puede influir en este escenario conociendo este contexto y usándolo como recurso para promover salud. El escenario individual se puede influir a través de la motivación hacia estilos de vida saludables desde los encuentros con los prestadores de salud.

\section{CONSEJERÍA Y EDUCACIÓN DE LOS PACIENTES PARA PROMOVER ESTILOS DE VIDA SALUDABLES}

Es deber de los sistemas de salud promover cambios de estilo de vida en los pacientes y ponerlos al tanto de la conexión que existe entre los factores de riesgo y la salud. La evaluación de los factores de riesgo y la consejería deben ayudar a los pacientes a la adquisición de información, motivación y habilidades para mantener comportamientos saludables ${ }^{(4)}$.

\section{CONSEJERÍAS RECOMENDADAS}

Dieta. Los mensajes que deben incorporarse en la consejería nutricional deben incluir el consumir alimentos variados en las tres comidas principales, y dos adicionales, para mantenerse saludables; una porción diaria de alimentos de origen animal, tres porciones de lácteos hasta alcanzar un equivalente entre 1000 a $1500 \mathrm{mg}$ de calcio al día; tres porciones de verduras crudas o cocidas, en variedad de colores; limitar la ingesta de sodio; consumir 6 a 8 vasos al día de agua hervida sin azúcar para mantenerse hidratado. Si hay problemas de dentición consumir alimentos blandos o triturados en forma de purés. Recordar que una alimentación variada no necesita de ningún tipo de suplementación adicional ni vitamínica ${ }^{(5)}$

Ejercicio. El ejercicio beneficia a personas de todas las edades, puede disminuir todas las causas de morbilidad e incrementar la expectativa de vida ${ }^{(6)}$. Entre los beneficios del ejercicio en el adulto mayor se incluyen la mejora del acondicionamiento, reduce el riesgo de enfermedad cardiovascular, enfermedad cerebro vascular, hipertensión, diabetes mellitus tipo 2, osteoporosis, obesidad, cáncer de colón, cáncer de mama, ansiedad, depresión, deterioro cognitivo y, finalmente, reduce el riesgo de caídas y lesiones. La evidencia sugiere que los beneficios del ejercicio, incluyendo la disminución de la mortalidad y mantenimiento de la independencia, ocurre con la iniciación, así como con la continuidad del ejercicio, incluso en adultos mayores de entre 75 y 85 años ${ }^{(6)}$.

Toda población sedentaria debe ser estimulada para desarrollar el hábito de la actividad física regular. Se debería dar una prescripción breve de la recomendación de ejercicios de acuerdo al estado de salud y los estilos de vida del paciente. Las recomendaciones incluyen de 20-30 minutos de actividad aeróbica como caminatas y trotes, entre 3 y 5 días por semana; ejercicios para mantener o incrementar la masa muscular, que deben incluir entrenamiento con pesas; ejercicios de calistenia con pesas o ejercicios de resistencia, y actividades que ejercitan el equilibrio, recomendadas para mejorar la estabilidad y prevenir las caídas y problemas relacionadas a ellas. Por otro lado, los ejercicios de balance dinámico, tal como el tai chi, son los más accesibles y no necesitan estricta supervisión.

Consejería sobre enfermedades de transmisión sexual. Los adultos mayores sexualmente activos deben recibir consejería sobre sexo seguro y uso de preservativos con nuevos compañeros sexuales. Ante conductas de riesgo se debe propiciar consejería para cambio de conducta, de preferencia en varias sesiones, especialmente en adultos mayores sexualmente activos y con riesgo incrementado para ITS ${ }^{(7)}$.

Tabaquismo. A cualquier edad en que se cese el tabaquismo se obtiene beneficio para la salud. Se debe preguntar a todos los adultos mayores si fuman, la periodicidad mínima de la detección del consumo debe practicarse, como mínimo, cada 2 años. Ante una respuesta positiva se debe intervenir para suspender el consumo de tabaco (Tabla 1). Se ha detectado diversas técnicas para suspender el uso del tabaco, ello incluye la recomendación del médico, la consejería formal y la farmacoterapia $^{\left({ }^{(8)}\right.}$ (Tabla 1).

Uso de alcohol. Aproximadamente $15 \%$ de los adultos mayores por encima de los 65 años experimentan problemas de salud relacionados con las complicaciones 
del consumo de alcohol en combinación con la medicación o con enfermedades crónicas. En 2 a $4 \%$ se encuentra criterios para alcoholismo. El uso de alcohol en el adulto mayor puede impactar negativamente en la función y la cognición, así como en la salud en general ${ }^{(9)}$.

La US Preventive Service Task Force (USPSTF) recomienda el tamizaje e intervención conductual en adultos en el entorno de la atención primaria para reducir el consumo de alcohol. La Sociedad Americana de Geriatría recomienda preguntar anualmente acerca del uso de alcohol indagando acerca de la frecuencia y cantidad de consumo, y luego aplicar el cuestionario CAGE (10) para hacer el diagnóstico precoz de problemas relacionados al consumo de alcohol (C: ¿ $\mathrm{Ha}$ sentido alguna vez que debe beber menos? A: ¿Le ha molestado que la gente lo critique por su forma de beber? G: ¿Alguna vez se ha sentido mal o culpable por su forma de beber? E: ¿Alguna vez ha necesitado beber por la mañana para calmar los nervios o eliminar molestias por haber bebido la noche anterior?).

\section{PREVENCIÓN DE ENFERMEDAD EN EL ADULTO MAYOR}

Para la realización de actividades preventivas en adultos mayores, se deben tener en cuenta diversos aspectos; considerando no solo el riesgo de enfermedad sino las espectativas, ideas, sentimiento y alteración de función que produce la enfermedad y el contexto particular de cada persona.

\section{MEDIDAS DE PREVENCIÓN PRIMARIA}

\section{INMUNIZACIONES EN EL ADULTO MAYOR}

El calendario de vacunaciones para los mayores de 60 años vigente en EE. UU. aprobado por el Centro para el Control y Prevención de Enfermedades (CDC), incluye cuatro vacunas: antitetánica/difteria, vacuna contra el herpes zoster, vacuna contra la influenza y la vacuna antineumocócica El calendario de vacunaciones del MINSA para adultos mayores incluye la vacuna contra la influenza ${ }^{(11)}$.

\section{MEDIDAS DE PREVENCIÓN SECUNDARIA}

\section{TAMIZAJE DE CÁNCER DE MAMA}

Cerca de la mitad de los casos de cáncer de mama son detectados a partir de los 65 años. El autoexamen de mama no ha demostrado efectividad en la disminución de mortalidad por cáncer, por esto, debería realizarse en el contexto de la consulta; con médicos entrenados ${ }^{(12)}$.

La mamografía también es controversial ya que los estudios que la validan como prueba de tamizaje, han incluido pocas mujeres mayores de 75 años. Sin embargo, tiene eficacia demostrada en disminuir mortalidad en las mujeres entre 50 y 69 años; la USPTF recomienda el cese del tamizaje a los 70 años; no obstante, a partir de los 70 años, algunos expertos recomiendan su uso bianual en mujeres con una buena expectativa de vida ${ }^{(13)}$.

\section{TAMIZAJE DE CÁNCER DE CUELLO UTERINO}

Una proporción significativa de mujeres adultas mayores nunca han tenido un Papanicolaou. Las mujeres que son, o han sido, sexualmente activas, deberían tener un Papanicolaou por lo menos cada 3 años, hasta los 65 años. A partir de los 65 años, si la mujer ha tenido tres tamizajes consecutivos negativos, idealmente incorporada en un programa de detección, se puede cesar el tamizaje ${ }^{(13)}$. A las mujeres mayores de 65 años sin citología en los últimos 5 años se les puede ofrecer dos citologías con periodicidad anual y, si son normales, no se propondrán más intervenciones. Sin embargo, en mujeres con edades por encima de los 70 años y sin Papanicolaou previo habrá que tener en cuenta las circunstancias individuales, tales como la expectativa de vida, capacidad de soportar el tratamiento si el cáncer es detectado y la posibilidad de cooperar y tolerar con el procedimiento de toma del Papanicolaou.

\section{TAMIZAJE DE CÁNCER COLORRECTAL}

Existe evidencia a favor del tamizaje para cáncer colorrectal sólida. La realización de la prueba de sangre oculta en heces es una medida que ha demostrado disminuir la mortalidad por cáncer de colon en $33 \%$, además de ser la prueba más costo-efectiva; pero, en adultos mayores tiene una mayor tasa de falsos positivos ${ }^{(14)}$. Son opciones

Tabla 1. Abordaje del tabaquismo: intervención mínima estructurada. Modelo de las 5A adaptado de la OMS

\begin{tabular}{ll}
\hline Averiguar (Ask) & $\begin{array}{l}\text { Identificar el consumo de tabaco en } \\
\text { cada consulta, grado de dependencia, } \\
\text { intentos previos, motivos de recaídas. }\end{array}$ \\
Aconsejar (Advise) & $\begin{array}{l}\text { Recomendar con firmeza la cesación } \\
\text { de fumar, hablar sobre los prejuicios } \\
\text { del tabaco y las ventajas de su } \\
\text { abandono. Resolver dudas. }\end{array}$ \\
Apreciar (Asses) & $\begin{array}{l}\text { Valorar la disponibilidad de abandonar } \\
\text { el consumo de tabaco, intervenir } \\
\text { según la etapa de cambio. }\end{array}$ \\
\hline Ayudar (Assist) & Ofrecer un plan concreto de ayuda. \\
\hline $\begin{array}{l}\text { Acordar y programar } \\
\text { (Arrange) }\end{array}$ & $\begin{array}{l}\text { Establecer visitas de seguimiento } \\
\text { específicas. }\end{array}$ \\
\hline
\end{tabular}


de tamizaje con eficacia similar, aunque con mayor costo según su complejidad: la prueba de sangre oculta en heces anual, la sigmoidoscopía flexible cada 5 años, y la colonoscopía cada 10 años.

Dada su capacidad para visualizar todo el colón, la colonoscopía podría constituirse en el método invasivo de elección; sin embargo, acarrea un riesgo incrementado en el adulto mayor. Según la USPSTF el tamizaje con cualquiera de estas opciones debe recomendarse desde los 50 hasta los 75 años. En aquellos pacientes con enfermedades que dificulten el procedimiento o con pobre estado funcional, en quienes se espera que vivan lo suficiente como para beneficiarse del tamizaje, podría considerarse la tomografía computarizada colonográfica.

\section{TAMIZAJE DE CÁNCER DE PRÓSTATA}

Aunque la Sociedad Americana de Cáncer recomienda el examen rectal digital y dosaje del PSA sérico anualmente, si la expectativa de vida es $\geq 10$ años, estas pruebas no son recomendadas por la USPTF como pruebas de tamizaje, pues aunque el PSA detecta cáncer en estadios más tempranos, no parece disminuir la mortalidad por esta causa, ni mejorar los resultados en salud.

Debe tenerse en cuenta que el PSA se eleva en adultos mayores no solo por cáncer sino por hiperplasia benigna de próstata. Asimismo, recordar que los hombres entre 65 y 70 años tienen mayor posibilidad de morir por una comorbilidad que por el cáncer prostático. En general, los mayores de 70 años y aquellos con una expectativa de vida inferior a 10 años no se benefician del cribado. Es por esto que, con excepción de las personas que lo soliciten y habiendo explicado los riesgos y beneficios del tamizaje, este no se recomienda en adultos mayores como rutinario ${ }^{(15)}$.

\section{TAMIZAJE DE GLAUCOMA}

La Academia Americana de Oftalmología recomienda una evaluación concienzuda de los ojos cada 1 a 2 años en los mayores de 65 años ${ }^{(16)}$. No existe evidencia sólida para recomendar a favor o en contra del tamizaje de glaucoma en adultos. Aunque, en base a opiniones de expertos, se recomienda evaluar rutinariamente la agudeza visual y hacer una evaluación oftalmológica integral anual a partir de los 65 años ${ }^{(17)}$.

\section{TAMIZAJE DE HIPERTENSIÓN}

La hipertensión arterial es altamente prevalente entre los adultos mayores y se mantiene como un factor de riesgo subyacente para padecer enfermedad isquémica cardiaca y stroke. La USPSTF, el Grupo de Trabajo de Servicios Preventivos Canadienses (CTFPHC), ACP, AAFP, y la Sociedad Americana de Geriatría (AGS) recomiendan la evaluación periódica de la presión arterial con rangos que van de 1 a 2 años ${ }^{(18)}$

\section{TAMIZAJE DE HIPERLIPIDEMIA}

El Panel Americano de Educación para el Colesterol (ATPIII) ${ }^{(19)}$ recomienda el tamizaje a todos los adultos entre 30 y 65 años, después de esta edad no existen estudios contundentes sobre si el uso de medicación hipolipemiante disminuya los eventos cardiovasculares como medida de prevención primaria, por lo cual se recomienda el tamizaje con colesterol total y HDL colesterol a los adultos mayores con bajo riesgo cardiovascular que tengan una buena expectativa de vida. En el 2013 el Panel Americano de Educación para el Colesterol (ATP IV) menciona con respecto a la prevención primaria, que es razonable evaluar de manera tradicional los factores de riesgo para enfermedad cardiovascular (ECV) ateroesclerótica cada 4 a 6 años en individuos que tienen entre 20 y 79 años y que están libres de enfermedad ateroesclerótica, y evaluar el riesgo de ECV ateroesclerótica estimada a los 10 años (utilizando The Pooled Cohort Equations cuya calculadora se puede encontrar en http://my.americanheart.org/cvriskcalculator) cada 4 a 6 años en adultos entre 40 y 79 años ${ }^{(20)}$.

Los pacientes en prevención secundaria deberán ser cribados anualmente mediante una determinación plasmática de colesterol total colesterol HDL, colesterol-LDL y triglicéridos. Deberán recibir una adecuada intervención sobre hábitos dietéticos, consejo antitabaco y ejercicio físico junto con el manejo farmacológico pertinente.

\section{TAMIZAJE DE OSTEOPOROSIS}

Solo el $3 \%$ de las mujeres con fractura por osteoporosis recibieron alguna vez una evaluación con densitometría ósea. La densitometría ósea no se considera una buena técnica de cribado poblacional, siendo más importante la búsqueda selectiva de casos. Eleva su rendimiento cuando se realiza en personas que presentan varios factores de riesgo para fractura (antecedente familiar, IMC, raza, tabaquismo, uso de corticoides). Se recomienda el tamizaje con densitometría a todas las mujeres de 65 años o mayores que decidan iniciar terapia farmacológica de encontrarse un resultado anormal y a todos los hombres mayores de 70 años bajo las mismas características ${ }^{(21)}$.

\section{MEDIDAS DE PREVENCIÓN CUATERNARIA}

La prevención cuaternaria se define como: "acciones o medidas adoptadas para identificar a un paciente en riesgo de medicalización excesiva, para protegerlo de 
nuevas intervenciones invasivas médicas, y sugerir intervenciones éticamente aceptables". Se basa en los cuatro principios éticos: autonomía, beneficencia, no maleficencia, justicia (22). Algunas acciones de prevención cuaternaria en adultos mayores incluyen el evitar la medicalización innecesaria, el sobrediagnóstico y el sobretratamiento, y tomar en cuenta las espectativas y deseos de los pacientes.

\section{PREVENCION DE FRAGILIDAD}

Otros de los aspectos que considerar para procurar la mejor condición de salud en la población que envejece, es abordar tempranamente la condición de fragilidad, entendida como el síndrome biológico de vulnerabilidad al cual se llega por el declive acumulado de los mecanismos fisiológicos, con pérdida progresiva de la capacidad homeostática, originando que el paciente no pueda enfrentar condiciones estresantes como infecciones, traumatismos, hospitalizaciones, entre otras. La importancia de la detección e intervención temprana de fragilidad, más aun, por su posibilidad de reversibilidad ${ }^{(23)}$, es la asociación de fragilidad con eventos no deseados en la población adulta mayor, como son: pérdida de función, discapacidad, caídas, progresión de comorbilidad, mala calidad de vida, hospitalizaciones, institucionalización y muerte (23); el identificar fragilidad permite medir el riesgo a desenlaces adversos, caracterizando el estado funcional y de salud más allá de la suma de enfermedades y discapacidad. Existe una amplitud de mecanismos causales de fragilidad, justificando la necesidad de intervenir en varios aspectos, para conseguir el objetivo de prevenir fragilidad.
Existen diferentes criterios para diagnosticar fragilidad, los más usados son los de Fried, (fenotipo de fragilidad) ${ }^{(23)}$, que operativiza la pérdida de peso no intencional, la debilidad muscular, la baja resistencia (cansancio), la lentitud de la marcha y el nivel bajo de actividad física; considerando frágil al paciente con 3 o más criterios, prefrágiles a quienes tienen 1-2 y robustos (no frágiles) a quienes no tienen ninguno de ellos. Otra propuesta interesante, es el "Instrumento de Fragilidad para la Atención Primaria de la Encuesta de Salud, Envejecimiento y Jubilación en Europa" SHARE-FI, que adapta los criterios de Fried y Waltson y cuenta con una calculadora de acceso libre disponible en https://sites.google.com/a/tcd.ie/share-frailty-instrumentcalculators/ (23). Otra forma rápida, económica y fácil de reproducir, que evalúa el aspecto funcional de fragilidad, es la velocidad de marcha (tiempo en recorrer a ritmo habitual una distancia establecida), el punto de corte marcador de fragilidad es menos de $1 \mathrm{~m} / \mathrm{s}^{(26)}$. Considerando el sentido causal multifactorial, una herramienta que sistematiza la evaluación del adulto mayor y permite acercar a los factores condicionantes de fragilidad, además de ser guía para estructurar medidas de intervención en base a los problemas, es, sin lugar a duda, la valoración geriátrica integral (VGI) ${ }^{(25-27)}$; una aproximación al VGI se busca con el índice clínico de fragilidad, escala que evalúa: actividades básicas e instrumentales, marcha y equilibrio, estado nutricional y cognición ${ }^{(28)}$.

En este contexto, proponemos estrategias y medidas de intervención para la prevención de fragilidad desde la perspectiva de atención primaria (Figura 1).

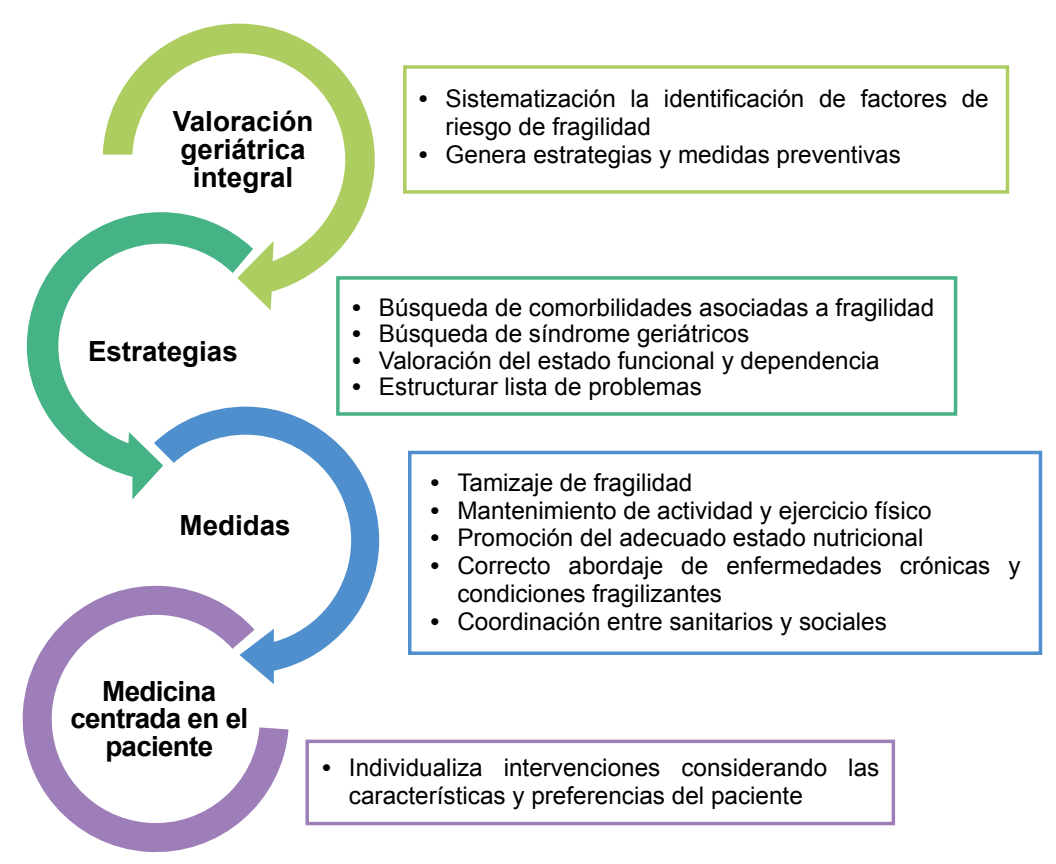

Figura 1. Prevención de fragilidad desde la perspectiva de atención primaria ${ }^{(26,27)}$ 


\section{Estrategias para prevención de fragilidad}

1. Búsqueda de comorbilidades asociadas a fragilidad; permitiendo el diagnóstico oportuno de comorbilidades y planificar su control adecuado.

2. Búsqueda de síndromes geriátricos. Facilita la identificación e intervención, poniendo especial interés en los que originan dismovilidad (dificultades para el movimiento) y riesgo nutricional.

3. Valoración del estado funcional y dependencia. Permite la estimación del estado funcional y el grado de dependencia para actividades básicas e instrumentales de vida diaria, estimando las condiciones de posible reversibilidad y procurando la búsqueda del soporte sociosanitario necesario para suplir las dependencias que propiciaran la aparición y progresión de fragilidad, como son la ayuda para alimentación adecuada, la higiene y los traslados.

4. Estructurar una lista de problemas, priorizando las condiciones que ocasionan fragilidad y factores desencadenantes. Facilita el diseño de un plan de trabajo multidisciplinario, coordinado; que contenga todas las medidas a tomar para revertir prefragilidad, prevenir fragilidad y estructurar el adecuado y oportuno seguimiento.

\section{MEDIDAS DE INTERVENCIÓN PREVENTIVA DE FRAGILIDAD EN ATENCIÓN PRIMARIA}

\section{TAMIZAJE DE FRAGILIDAD}

Buscar fragilidad mediante alguna herramienta mencionada $u$ otra, indicado a todos los mayores de 80 años, y entre 65 y 80 años entre pacientes con condiciones de riesgo. Complementar la evaluación con valoración geriátrica integral a pacientes con factores de riesgo y prefrágiles ${ }^{(26)}$.

\section{MANTENIMIENTO DE LA ACTIVIDAD Y PROMOCIÓN DEL EJERCICIO FÍSICO}

Las actividades de consejería son necesarias para potenciar aspectos de movilidad, capacidad física y disminución de riesgos; generar recomendaciones que propicien la actividad y, de ser posible, prescribir ejercicio físico de cierta intensidad, adecuado a las características de cada persona, en coordinación con fisioterapia. Son útiles los programas de entrenamiento de fuerza con resistencia progresiva, que están demostrando tener un importante papel en prevenir fragilidad y revertir prefragilidad $^{(25-27,29)}$.

\section{EVALUACIÓN Y PROMOCIÓN DE UN ADECUADO ESTADO NUTRICIONAL}

Evaluar un estado nutricional adecuado que garantice la reserva proteica, calórica y de oligoelementos en niveles apropiados, promoviendo la ingesta de una dieta balanceada con buen aporte proteico: $1-1,5 \mathrm{gr} / \mathrm{kg} / \mathrm{día}$, distribuido en al menos tres tomas; calórico: $30-38 \mathrm{kcal} /$ $\mathrm{kg} / \mathrm{día}$, así como de micronutrientes entre ellos vitamina $\mathrm{D}$; adecuando las cantidades y regulando volúmenes, para compensar el gasto energético de las actividades y en algunas circunstancias compensando gastos adicionales ocasionados por enfermedades crónicas. Es importante que las dietas restrictivas prescritas por enfermedades crónicas sean evaluadas con cautela y evitar que estas ocasionen pérdida de peso brusca y, de ninguna manera, sarcopenia por su demostrada asociación con fragilidad ${ }^{(30)}$. Por otro lado, se debe estar atento y evitar limitaciones por otras circunstancias como: fármacos anorexígenos, dependencia funcional, salud oral, condiciones socioeconómicas y culturales. Se deben gestionar actividades que faciliten educación sobre dieta equilibrada y suficiente, así como programar seguimiento periódico de medidas de intervención realizadas. La administración de los suplementos puede ser de utilidad en las personas de edad con desnutrición, restricción de volumen y otras circunstancias específicas ${ }^{(25,26,31)}$.

\section{CORRECTO ABORDAJE DE ENFERMEDADES CRÓNICAS Y CONDICIONES FRAGILIZANTES}

Una medida importante, es la oportuna identificación y correcto tratamiento individualizado de condiciones como: artrosis, cáncer, diabetes, deterioro cognitivo, demencia, depresión, enfermedad cardiovascular, enfermedad renal crónica, EPOC, osteoporosis, malnutrición, deprivación neurosensorial, dolor, incontinencia, inestabilidad de la marcha, caídas, síndrome confusional agudo, y riesgo social $(23,25,27)$. El abordaje de estas condiciones deberá estar orientado a su búsqueda y control periódico con medidas de intervención que den espacio a la educación, permitiendo al paciente conocer sus enfermedades, dándole la oportunidad para tomar decisiones y optimizar adherencia al tratamiento; tener como objetivo la recuperación y, si es posible, evitar exacerbaciones y complicaciones; prevenir hospitalizaciones y, sobre todo, priorizar el mantenimiento de la funcionalidad. En muchas circunstancias los objetivos terapéuticos tendrán que ser ajustados, atendiendo consideraciones personales propias de las características individuales de la enfermedad en el paciente adulto mayor y ejerciendo medicina centrada en el paciente, que es la mejor estrategia para conseguir objetivos terapéuticos, evitando polifarmacia y prescripciones inadecuadas $(25,27)$. 


\section{COORDINACIÓN ENTRE AGENTES SANITARIOS Y SOCIALES}

Al ser muchos los condicionantes de fragilidad y muchos los problemas en los que intervenir, para prevenir serán necesarios algunos profesionales, no siempre disponible en un mismo lugar, por lo que será absolutamente necesario estructurar una red que trabaje coordinadamente, mantenga comunicación fluida y esté conformada por profesionales capacitados, experimentados en problemas de salud del adulto mayor, con visión integral y experiencia de trabajo en equipo; al ser los médicos familiares y equipos del primer nivel los primeros en entrar en contacto con el paciente, de acuerdo al caso, será necesaria la coordinación con: geriatras, rehabilitadores, fisioterapeutas, nutricionistas, asistentes sociales, odontólogos, oftalmólogos, otorrinolaringólogos,

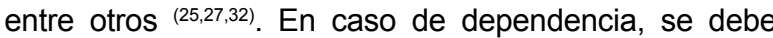
promover un adecuado soporte social, facilitando la distribución de los recursos, dando apoyo educacional y emocional al cuidador, integrándolo al equipo de cuidado.

Las enfermedades y condiciones fragilizantes se van acumulando con el trascurso de los años, evitar o intervenir solo en una de ellas de forma aislada, no evita el riesgo de sufrir cualquier otra, ni evitará desarrollar fragilidad. Los tratamientos vigentes centrados en la enfermedad, con abordaje terapéutico de uno a la vez, han demostrado no ser eficaces y menos en poblaciones de edad avanzada; en esa perspectiva radica la importancia de detectar fragilidad, independiente de enfermedad y discapacidad, porque nos ofrece la oportunidad de intervención precoz y así evitar todos los eventos no deseados mencionados, para una adecuada condición de salud del adulto mayor.

\section{PREVENCIÓN DE CAÍDAS}

Las caídas son una condición frecuente en el adulto mayor, 30 a $60 \%$ de los adultos mayores de la comunidad sufren un episodio de caída al año ${ }^{(34)}$. Su incidencia anual se aproxima a $50 \%$ en pacientes por encima de 80 años. El $5 \%$ de caídas en los adultos mayores resulta en fracturas u hospitalización (35). Según el Panel of Prevention of Falls in Older Persons: La caída es un evento caracterizado por la perdida de la estabilidad postural con un desplazamiento del centro de gravedad hacia un nivel inferior, generalmente el suelo, sin una previa perdida del conocimiento y sin perdida del tono postural, ocurriendo de una manera no intencional (35). Muchas veces se asume las caídas como algo propio del proceso del envejecimiento, sin embargo, un episodio de caída no es una enfermedad en sí misma, pero puede ser la manifestación de múltiples enfermedades tanto agudas como crónicas, lo que se suma a los cambios que ocurren propios del envejecimiento (36).
A pesar de que las caídas son una condición frecuente en el adulto mayor, en muchas ocasiones es difícil detectarlo, ya que no solo no son observadas por el profesional de salud o por la familia del paciente, sino por que muchas veces es atribuido al proceso del envejecimiento. Es por ello que el Instituto Nacional para la Excelencia Clínica (NICE) propone interrogar a todos los adultos mayores acerca de caídas sufridas. Si estos tuviesen un riesgo incrementado, es decir, que reporten al menos dos caídas en el año previo o una caída con lesión, además de la evaluación y manejo de causas subyacentes, debería brindársele información acerca de medidas que tomar para evitar caídas en el futuro, referirlos hacia programas con estrategias de prevención de caídas, explicarles la naturaleza prevenible de un episodio de caída, así como los beneficios físicos y psicológicos de modificar el riesgo de caídas (como la pobre iluminación, barreras arquitectónicas); debe, así mismo, brindarse información de cómo actuar ante un episodio de caída y la importancia de evitar la permanencia por largo tiempo en el suelo ${ }^{(34)}$.

\section{EVALUACIÓN DEL RIESGO DE CAIIDAS}

Dada la naturaleza multifactorial de las caídas, la evaluación de los factores de riesgo para los adultos mayores que presentan caídas o que tengan algún trastorno de la marcha o el equilibrio debe de ser multifactorial.

El profesional de salud, al ponerse en contacto con el adulto mayor, debería de evaluar el riesgo de caídas, para ello, el Panel en Prevención de Caídas en las personas mayores recomienda realizarlo a través de tres preguntas: ¿tuvo dos o mas caídas en el ultimo año?, ¿tuvo una caída reciente?, ¿tiene dificultad para caminar o del equilibrio? Por otro lado, el profesional de salud, especialmente los de atención primaria, es el que establece el primer contacto con el adulto mayor de la comunidad, por lo que debería conocer sobre qué medidas tomar frente a un adulto mayor con riesgo de caídas (Figura 2) ${ }^{(35,36) .}$

\section{INTERVENCIONES PARA LA PREVENCIÓN DE CAÍDAS}

Existen diferentes tipos de intervenciones preventivas, sin embargo, se sabe que las intervenciones multifactoriales y multicomponente son las más efectivas para la disminución del riesgo de caídas. Se denomina intervención multicomponente a las que ofrecen más de una intervención y están dirigidas a toda la población específica. Por otro lado, las intervenciones multifactoriales están orientadas a la población de la comunidad y se dan según la valoración previa de factores de riesgo. Ambas intervenciones tienen como pilar al ejercicio físico, la evaluación médica, la modificación del entorno y el ajuste de la medicación (36). 


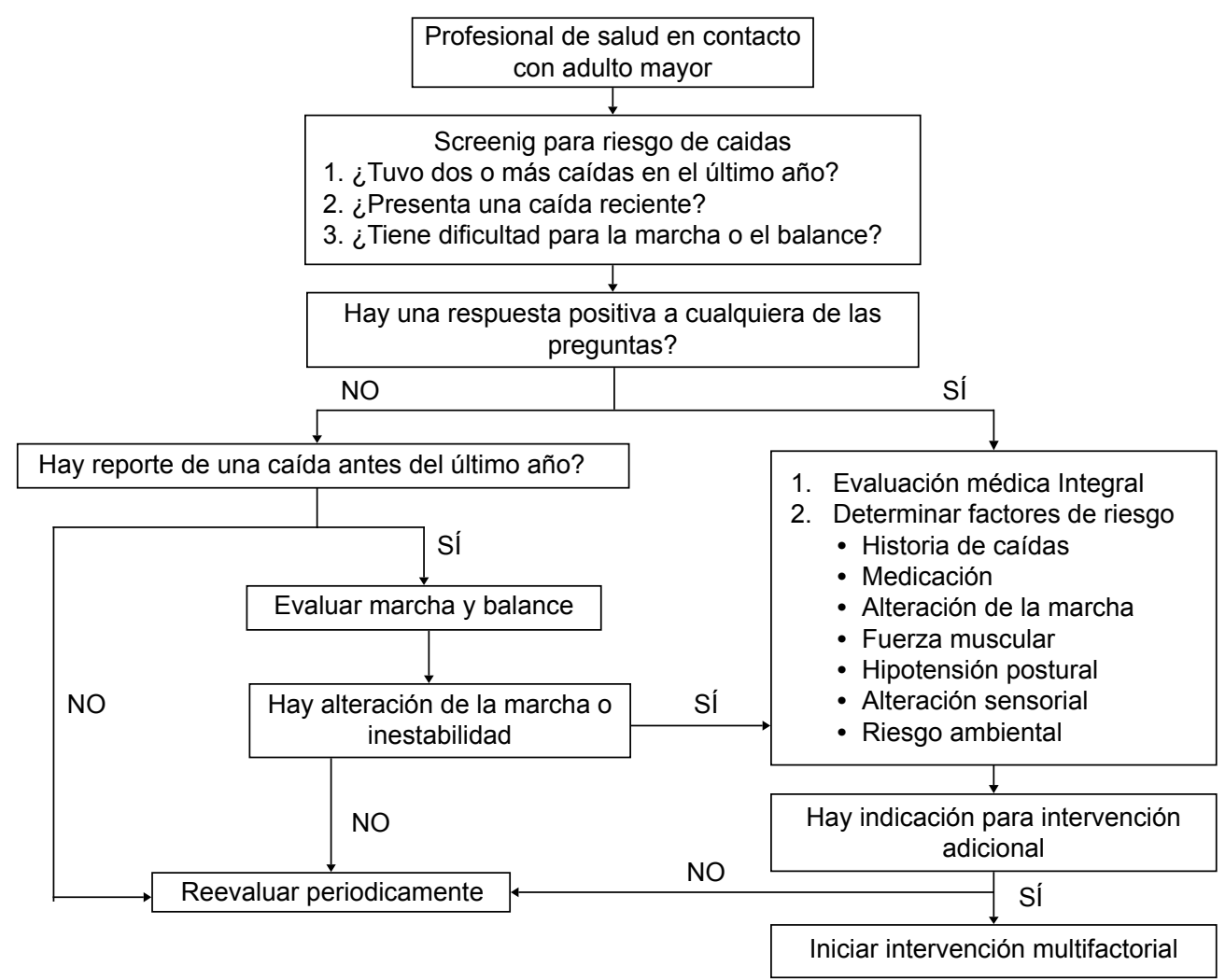

Figura 2. Prevención de caídas en el adulto mayor de la comunidad (36)

El ejercicio físico es la intervención más beneficiosa y las más probada en la prevención de caídas. Existen diversos tipos de entrenamiento como el de fuerza, resistencia aeróbica, equilibrio, y multicomponente. Sin embargo, son el entrenamiento multicomponente y el taichí (realizados en grupo) los que ofrecen mayor beneficio en la reducción de la tasa y riesgo de caída. El entrenamiento de equilibrio debe incluir ejercicios en tándem y semitándem, desplazamiento en múltiples direcciones con pesos extra, y caminar con apoyo talón punta. Con respecto al entrenamiento aeróbico, siempre debe realizarse asociado a ejercicios de equilibrio. De otro lado, el entrenamiento de fuerza es especialmente útil en adultos mayores frágiles. Por concenso, la duración y frecuencia del entrenamiento es de tres sesiones semanales, estas deberían de sumar dos horas a las semana, durante un periodo de 3 a 6 meses ${ }^{(25)}$. Así mismo, es importante señalar que mientras mas gradual sea la progresión del entrenamiento, mejor será la tolerancia a este, y menor será los efectos secundarios.

Se sabe que la polifarmacia, y el uso de algunos fármacos, en particular los psicotrópicos, incrementan el riesgo de caídas y fracturas hasta en un $81 \%(36,37)$. Por ello, en un adulto mayor que se cae, o con riesgo de caídas, debe disminuir la polimedicación, y valorar el retiro progresivo de fármacos que aumenten en riesgo de caídas como los antidepresivos tricíclicos, inhibidores de la receptación de serotonina, benzodiacepinas de vida media larga y corta, y los psicotrópicos típicos y atípicos, el retiro gradual de antipsicóticos reduce el porcentaje de caídas ${ }^{(36)}$.

La modificación del entorno, que consiste en disminuir las circunstancias y los objetos que favorecen las caídas fuera y dentro del domicilio, constituye una estrategia eficaz que disminuye el porcentaje de caídas y el riesgo de caídas, pero en adultos mayores de alto riesgo con déficit visual importante ${ }^{(36)}$. Otras intervenciones incluyen la corrección del déficit visual, el manejo de la hipotensión ortostática, el manejo de los problemas de los pies, y las intervenciones dirigidas a tratar morbilidades específicas que causan caídas. Por ejemplo, las caídas por hipersensibilidad del seno carotideo requieren marcapaso ${ }^{(36)}$.

\section{PREVENCIÓN DE COMPLICACIONES IATROGENICAS}

Las complicaciones iatrogénicas son más comunes y más graves en los adultos mayores, e incluyen los eventos adversos a medicamentos, infecciones nosocomiales, úlceras por presión, delirio, entre otras. Es importante identificar a pacientes en riesgo como aquellos pacientes con pluripatología (a mayor número de enfermedades crónicas mayor riesgo de que el tratamiento de una 
enfermedad exacerbe a las demás); pacientes tratados con múltiples médicos (que genera una atención no coordinada y polifarmacia); con polifarmacia (aumenta el riesgo de interacciones adversas), y pacientes hospitalizados (la hospitalización aumenta el riesgo de infecciones nosocomiales, polifarmacia, entre otros). En este sentido, las intervenciones que han demostrado prevenir complicaciones iatrogénicas son: la evaluación por equipo geriátrico interdisciplinario, el manejo en una unidad de cuidados de agudos geriátricos y, finalmente, orientar al paciente sobre directrices avanzadas ${ }^{(35,36)}$.

\section{CONCLUSIÓN}

En resumen, las actividades de promoción de la salud y prevención de las enfermedades en el adulto mayor, buscan lograr un envejecimiento exitoso. Con respecto a las actividades de promoción de la salud, estas deben ser abordadas considerando el escenario macrosocial, microsocial, interpersonal e individual. De otro lado, las actividades preventivas en el adulto mayor deben estar dirigidas no solo al riesgo de enfermar, sino a la alteración de la función que produce la enfermedad, y otras condiciones que puedan deteriorar la salud del adulto mayor, como la fragilidad, las caídas, y las complicaciones iatrogénicas. Es importante dar al adulto mayor las pautas necesarias para ejercer un mejor control sobre su salud y mejorarla, intentando mantener en todo momento su autonomía, su calidad de vida y, sobre todo, respetando siempre sus valores y preferencias.

Contribuciones de autoría: EAD, SCF Y MMM han participado en la concepción del artículo. EAD, SCF Y MMM realizaron la redacción del artículo. EAD, SCF Y MMM hicieron revisión crítica del artículo.

Fuentes de financiamiento:autofinanciado.

Conflictos de interés: los autores declaran no tener conflictos de interés

\section{REFERENCIAS BIBLIOGRÁFICAS}

1. Organización Mundial de la Salud. Constitución de la Organización Mundial de la Salud: Principios. $48^{\mathrm{a}} \mathrm{Ed}$. Ginebra: OMS; 2014.

2. Organización Panamericana de la Salud. Carta de Ottawa para la Promoción de la Salud. Ottawa: OPS; 1986.

3. Glanz K, Rimer BK, Viswanath K. Helath behavior and health education. Theory, Research and Practice. $4^{a}$ Ed. San Francisco: WILEY; 2002

4. Richardson JP. Consideration for health promotion and disease prevention in older adult. California: Medscape; 2006.

5. OMS/FAO. Dieta, Nutrición y Prevención de Enfermedades Crónicas. OMS, Serie de Informes Técnicos, 916. Ginebra; OMS; 2013.

6. Howe TE, Rochester L, Neil F, Skelton DA, Ballinger C. Exercise for improving balance in older people. Cochrane DatabaseSyst Rev.2011;(11):CD004963. doi: $\quad 10.1002 / 14651858 . C D 004963$. pub3.

7. U.S. Preventive Services Task Force. The Guide to Clinical Preventive Service 2015, Recommendations of the U.S. Services Task Force. New York: Agency for Healthcare Research and Quality; 2015.

8. Larzelere MM, Williams DE. Promoting smoking cessation. Am Fam
Physician 2012;85(6):591-8.

9. Sally SK. Alcoholism in the elderly. Am Fam Physician. 2000;61(6):1710-6.

10. Ewing JA. Detecting alcoholism. The CAGE questionnaire. JAMA. 1984;252(14):1905-7. doi:10.1001/ jama.1984.03350140051025.

11. Ministerio de Salud. Prevención para la salud: Estrategia Inmunizaciones. Disponible en : http://www.minsa. gob.pe $/$ index.asp op $=2 \&$ box $=2$

12. Walter LC; Lewis CL; Barton MB. Screening for colorectal, breast, and cervical cancer in the elderly: a review of the evidence. Am J Med. 2005;118(10):1078-86. doi: http://dx.doi.org/10.1016/j. amjmed.2005.01.063

13. Iarc.fr [internet]. Lyon: International Agency for Research Cancer; 2016 [Actualizado 2010 Jun 15; citado 2010 Jul 12]. Disponible en: http:// screening.iarc.fr/cervicalindex.php

14. U.S. Preventive Services Task Force. Screening for Colorectal Cancer: U.S. Preventive Services Task Force. Recommendation Statement. Ann Intern Med. 2008;149(9):627-37. doi:10.7326/0003-4819-149-9200811040-00243.

15. Dahm P, Neuberger M, Ilic D. Screening for prostate cancer: shaping the debate on benefits and harms. Cochrane Database
Syst Rev. 2013;(9):10.1002/14651858. ED000067. doi: org/10.1002/14651858. ED000067.

16. Heflin M. Geriatric Health Maintenance [internet]. Schmader K: Uptodate; 2016 [Actualizado 2009 Sep 28; citado 2016 May 10]. Disponible en: http://www.uptodate/ geriatrichealthmaintenance

17. U.S. Preventive Services Task Force. Glaucoma for Screening. Clinical summary of U.S. Preventive Services Task Force Recommendation. Rockville: The USPSTF; 2013. Disponible en: http:// www.uspreventiveservicestaskforce.org/ Page/Document/UpdateSummaryFinal/ glaucoma-screening

18. Gnanadesigan N, Fung CH. Quality Indicators for Screening and Prevention in Vulnerable Elders. J Am Geriatr Soc. 2007;55 Suppl 2:S417-23. doi: 10.1111/j.1532-5415.2007.01350.x

19. Expert Panel on Detection, Evaluation, and Treatment of High Blood Cholesterol in Adults. ExecutiveSummary of The Third Report of The National Cholesterol Education Program (NCEP) Expert Panel on Detection, Evaluation, and Treatment of the High Blood Cholesterol in Adults (Adult Treatment Panel III). JAMA. 2001;16;285(19):2486-97.

20. A Report of the American College 
of Cardiology/American Heart Association Task Force on Practice Guidelines. 2013 ACC/AHA Guideline on the Treatment of Blood Cholesterol to Reduce Atherosclerotic Cardiovascular Risk in Adults. J Am Coll Cardiol. 2013;63(25):2889-934. doi:10.1016/j.jacc.2013.11.002

21. Williams C. Healthy aging and assession older adults. Southpaul J. Current Diagnosis and Treatment Family Medicine. 2a Ed. New York: Lange Medical Book; 2008. p. 433-51.

22. Miguel Pizzanelli. Prevención Cuaternaria, Un desafío Profesional, Clínico, ético y conceptual, para médicos del siglo XXI. Florida: Societat Balear de Medicina Familiar i Comunitaria; 2011.

23. Lee JS, Auyeung TW, Leung J, Kwok T, Woo J. Transitions in frailty states among community-living older adultsand their associated factors. J Am Med Dir Assoc. 2014;15(4):2816. doi: 10.1016/j.jamda.2013.12.002.

24. Romero-Ortuno R, Walsh CD, Lawlor BA, Kenny RA. A frailty instrument for primary care: findings from the Survey of Health, Ageing and Retirement in Europe (SHARE). BMC Geriatr. 2010;10:57. doi: 10.1186/1471-231810-57.

25. Abizanda-Soler P., Rodríguez-Mañas L. Fragilidad en el anciano. En: Tratado de medicina geriátrica. Fundamentos de la atención sanitaria a los mayores. España: ELSEVIER; 2014.
26. Abizanda P. Gomez J. Lesende I. Baztan J. Detección y prevención de la fragilidad: una nueva perspectiva de prevención de la dependencia en las personas mayores. Med Clin (Barc). 2010;135(15):713-9. doi: 10.1016/j. medcli.2009.04.028.

27. Rodriguez-Mañas L, Fried L. Frailty in the clinical scenario. Lancet. 2015;385(9968):e7-9. doi: 10.1016/ S0140-6736(14)61595-6.

28. Studenski S, Hayes RP, Leibowitx RQ, Bode R, Lavery L, Walston J, et al. Clinical global impresion of change in physical frailty: Development of a measure based on clinical judgement. J Am Geriatr Soc. 2004;52(9):15606. doi: 10.1111/j.15325415.2004.52423.x

29. Casas Herrero Á, Cadore EL, Martínez Velilla N, Izquierdo Redin M. El ejercicio físico en el anciano frágil: una actualización. Rev Esp Geriatr Gerontol. 2015;50(2):74-81. doi: 10.1016/j.regg.2014.07.003.

30. Carrillo R, Muciño J, Peña C, Carrillo U. Fragilidad y sarcopenia. Revista de la Facultad de Medicina de la UNAM. 2011; $54(5): 12$ 21.

31. Gaillard C, Alix E, Sallé A, Berrut G, Ritz P. Energy requirements in frail elderly people: A review of the literature. Clin Nutr 2007;26(1):1624. doi: 10.1016/j.clnu.2006.08.003

32. François Béland, Zunzunegu MV. Sistemas integrados de atención sanitaria al anciano frágil y dependiente. Tratado de medicina geriátrica. Fundamentos de la atención sanitaria a los mayores. España: ELSEVIER; 2014.

33. Rubenstein LZ, Josephson KR. The epidemiology of falls and syncope. Clin Geriatr Med. 2002;18(2):141-58

34. Varela L. Valoración geriátrica integral y síndromes geriátricos. En: Principios de geriatría y gerontología. $2^{\text {a }} \mathrm{Ed}$. Lima: Centro editorial Universidad Peruana Cayetano Heredia; 2011. p. 195- 207.

35. Abizanda-Soler P., Rodríguez-Mañas L. Caidas y trastorno de la marcha en el anciano. En: Tratado de medicina geriátrica. Fundamentos de la atención sanitaria a los mayores. España: ELSEVIER; 2014.

36. Panel on Prevention of Falls in Older Persons, American Geriatrics Society and British Geriatrics. Summary of the Update American Geriatrics Society/ British Geraitrics Society Clinical Practice guideline for Prevention of Falls in Olders Persons. J Am Geriatr Soc. 2011:59(1):148-157. doi: 10.1111/j.1532-5415.2010.03234.x.

Correspondencia: Elizabeth Aliaga Diaz

Dirección: Instituto de Gerontologia, Universidad Peruana Cayetano Heredia. Av. Honorio Delgado 430, Urbanización Ingeniería, San Martín de Porres Lima, Perú.

Teléfono: (+511) 987581800. Telefax: 319 0007 Anexo 2246

Correo electrónico: igero@oficinas-upch.pe, elizabeth.aliaga.d@upch.pe

Nuestros artículos se encuentran indizados en:

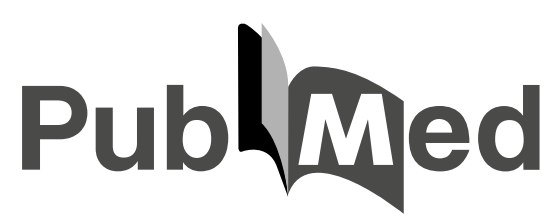

www.pubmed.gov 